\title{
Research on the Creative Tablet Folding Table
}

\author{
Ruihao Wang \\ Power System and Automation, School of North China Electric Power University \\ Baoding, Hebei, China \\ E-mail: 799309482@qq.com
}

\begin{abstract}
This paper first gives an analysis of the static structure and dynamic process of folding table, gets corresponding folding process parameter equation by geometric relations, and quantifies parameters respectively for different design requirements of desktop. The desktop projection just falls within the supporting surface, this time not only to meet the stability of the desktop, but also to guarantee load-bearing capacity of the desktop. By rotating planar projection method outermost strips of wood, reinforced strips of wood establish each position to the function of the distance and $\theta$ hinge. Similarly the establishment of legs on the edge point coordinates $\theta$ parameter equation, any legs folded edge line shape. By parameter equation can describe the dynamic changes of the folding table.In this paper the design scheme of two folding tables is given by 3DMAX and MATLAB.
\end{abstract}

Keywords_parameter optimization, stability, cosine theorem

\section{RESTATEMENT OF THE PROBLEM}

A company produces a folding table, with a circular desktop, and the table legs with hinge activities can be split into a tablet. The legs of the table by a plurality of wood composition are divided into two groups, each group with a reinforced wood connection. The two ends of the reinforcing steel bar are respectively fixed on the two sticks of most outside table legs in each group and along the wood there are empty slots to ensure the sliding degrees of freedom. The shape of the table is composed of a straight grain surface with a beautiful shape. The following issues are discussed in the establishment of a mathematical model

1)A given rectangular plate size of $120 \mathrm{~cm} \times 50$ $\mathrm{cm} \times 3 \mathrm{~cm}$, each root wood width $2.5 \mathrm{~cm}$, the battens connecting table legs are fixed in the center of the outermost wood of the table legs, the height of the table folded after is $53 \mathrm{~cm}$. The design parameters of the folding table and the mathematical description of the edge line of the table foot are presented.

2) The company plans to develop a folding table design software, to produce the desired shapes, according to the customers, for any set of folding table height, the edge of the desktop line size and shape of the legs of the table edge line rough shape, through giving the desired flat material, shape, size and feasible optimal design and processing parameters. The production of folding table can meet as far as possible the customer of their self-creative tables.

\section{Model eStablishment AND SOLUTION SELECTING}

\section{A.DESK TOP SIZE DETERMINATION}

First of all, the force of supporting legs of the table must be uniform. Whether it is by face or point for the support, the stability and safety are the most important. That requires the four table supporting legs must be completely symmetrical. Those with novel styles, but not practical, can only be called ornamentation. Because the shape and chair design is to follow its function, regardless of the breakthrough the shape, however, it cannot do without the support of the two surfaces, that is, supporting role.

\section{B. To Determine the Immersions of Wood}

Make the first strip of wood as a benchmark, then make a right triangle structure, by the geometry relationship, the expression of the length of the ith batten

$$
r_{i}=\sqrt{R^{2}-(25-2.5(\mathrm{i})-1)^{2}}
$$

From above the desktop parameters $r_{i}$, then ith batten of the table leg length can be determined 
$\mathrm{L}_{\mathrm{i}}=60-r_{i}$ Desktop size and table leg size is as follows:

\begin{tabular}{llcccc}
\multicolumn{5}{c}{ Table1 Desktop size } \\
\hline $\begin{array}{l}\text { sequence } \\
\text { number }\end{array}$ & $\mathbf{1}$ & $\mathbf{2}$ & $\mathbf{3}$ & $\mathbf{4}$ & $\mathbf{5}$ \\
\hline$r_{i}$ & 6.79 & 10.9 & 15 & 17.9 & 20 \\
$\mathrm{~L}_{\mathrm{i}}$ & 53.21 & 49.1 & 45 & 42.1 & 40 \\
\hline $\begin{array}{l}\text { sequence } \\
\text { number }\end{array}$ & 6 & 7 & 8 & 9 & 10 \\
\hline$r_{i}$ & 21.7 & 22.9 & 23.9 & 24.5 & 24.8 \\
$\mathrm{~L}_{\mathrm{i}}$ & 38.3 & 37.1 & 36.1 & 35.5 & 35.13 \\
& & & & & 10 \\
\hline
\end{tabular}

\section{Solutions and Dynamic Analysis of the Design} Parameters of the Folding Table

For the structure of the folding table, under the drive of the outside of the wood, the steel bar fixed at the center circles around the first hinge, and under the traction of steel bars, of the table legs rotate around the hinges on the desktop. In each folded state, all wood bars center respective hinges through reinforcement. So we can see this process decomposition, first determine the state of the rotation of the lateral batten, the location of the midpoint of the two pieces of wood with space between attachments is reinforced, and the midpoint of the line of two woods is the actual location of the wood. This process can be quantified, and it can be projected to the center $\mathrm{Y}-\mathrm{O}$ $Z$. The geometric relation in the projection shows that in the medium, cosine theorem can be used to solve the result, in the process of folding, reinforcing steel bar can move freely in the wood. For the dimensions of the slot $\Delta l_{i}$, namely slot size, and the specific calculation methods are as follows:

$$
\begin{aligned}
& a_{i}=r_{i}-r_{i-1} \\
& l_{i}=\sqrt{a_{i}^{2}+l_{1}^{2}-a_{i} l_{1} \cos \theta}
\end{aligned}
$$

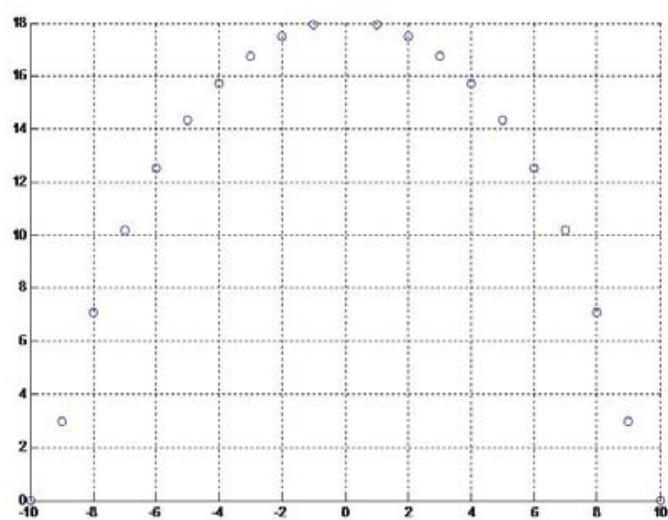

Figure 1: Schematic diagram of the wood slot length

13 By above knowable, the more the longer the slot length, near the center of basic is in the shape of parabola wood of the center slot length is $17.959 \mathrm{~cm}$, is apart from the edge of wood with $17.161 \mathrm{~cm}$.

As each folding state corresponding to a final state, slotted length will change according to the following rules:

$$
\Delta l_{i}=\sqrt{a_{i}^{2}+l_{1}^{2}-a_{i} l_{1} \cos \theta_{\max }}-\left(l_{1}-a_{i}\right) \quad i=1,2 \ldots 10 .
$$

When changes within $0^{\circ} \sim 90^{\circ}$ for final value, and when the corresponding change of is greater than $90^{\circ}$, $90{ }^{\circ}$ are taken

\section{The Tabletop Edge Line}

In the following coordinate system, the position of the bar and the position of the hinge are used to position the foot, and then the coordinates of the length of the end of the original are calculated. Take the second sticks as an example, for the final states of the folding, using the cosine theorem, the cos can be obtained:

$$
\cos \phi=\frac{a_{2}^{2}+l_{2}^{2}-l_{1}^{2}}{2 a_{2} l_{2}}
$$

The rotation law shows that they always cannot be rotated in the plane of $x=22.5$, by reinforcing bar positions and center of rotation can determine the position of wood 2, and is knows, therefore, depending on the geometry relations coordinates can be obtained as follows:

$$
\begin{aligned}
& y_{2}=-\left[\left(60-r_{2}\right)(-\cos \phi)+r_{2}\right] \\
& z_{2}=-\left(60-r_{2}\right) \sin \phi
\end{aligned}
$$

With the method above the edge coordinates of 20 wood sticks can be obtained, and then the terminal state of the table edge line is obtained.

Coordinate determination: 


$$
\begin{aligned}
& a_{i}=r_{i}-r_{i-1} \\
& \cos \phi_{i}=\frac{a_{i}^{2}+l_{i}^{2}-l_{1}^{2}}{2 a_{i} l_{i}} \\
& l_{i}=\sqrt{a_{i}^{2}+l_{1}^{2}-2 a_{i} l_{1} \cos \theta}
\end{aligned}
$$

From the above formula, the image of the edge of the foot can be obtained from different folding state, as is shown in Figure2.

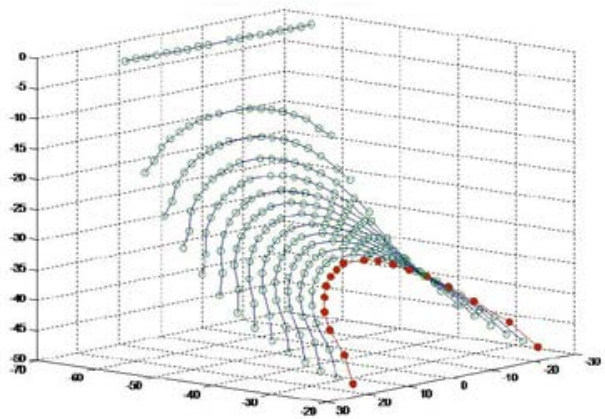

Figure2 Dynamic change process of the edge of the foot line

The red curve for the folding table final state of the legs of the table edge line, by coordinates equations can solve arbitrary folded state, and the spatial coordinates of each table leg edges get the edge of the state under the table foot shape. Therefore, in order to carry on the parameter equations of the spatial coordinates, the dynamic change of the edge of the table foot can be described.

III. The Design Of Creative Folding Table

With the three simply given requirements-- the height of the folding table, the shape and size of desktop edge line and the rough shape of the edge line of the table leg, the sheet size and related parameters such as the main support the position of the table leg, desktop every wood size (table leg length, desktop hinge position), reinforced position, and each of the wood slot length and related parameters should be determined. The methods of the former problem can be applied so as to determine the parameters.
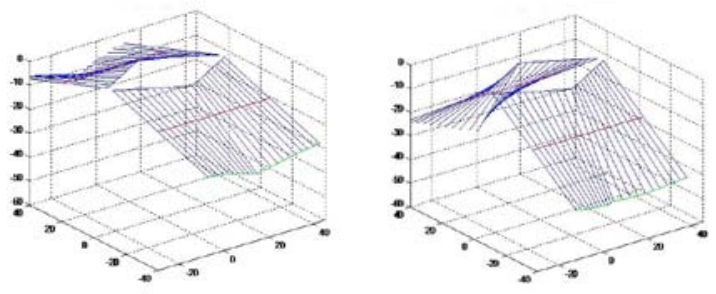
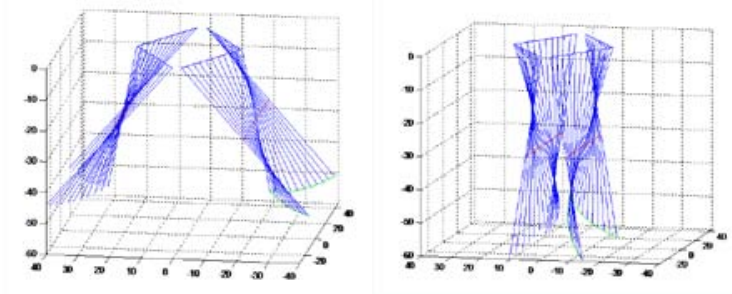

\section{REFERENCES}

[1] Jiang Qiyuan Xie Jinxing Ye Jun, "The Fourth Edition of Mathematics Modeling ", Higher Education Press, 2011

[2] Li Dingchong, "Additional Single Point Support Stability of Compressed Rod, Plate, the Influence of Computational Structural Mechanics and its Applications", vol. 4, 1 edi. February 1987

[3] Fang Quanxiao, "root in the soil and crop water quality model parameters optimization and uncertainty assessment," Journal of Agricultural Engineering, vol. 28, 10, May 2012 